\title{
Wireless Temperature Sensing with BST-Based Chipless Transponder Utilizing a Passive Phase Modulation Scheme
}

Mandel, Christian; Maune, Holger; Maasch, Matthias et al.

(2011)

$\begin{array}{ll}\text { DOI (TUprints): } & \text { https://doi.org/10.25534/tuprints-00014309 } \\ \text { Lizenz: } & \text { lediglich die vom Gesetz vorgesehenen Nutzungsrechte gemäß UrhG } \\ \text { Publikationstyp: } & \text { Artikel } \\ \text { Fachbereich: } & 18 \text { Fachbereich Elektrotechnik und Informationstechnik } \\ \text { Quelle des Originals: } & \text { https://tuprints.ulb.tu-darmstadt.de/14309 }\end{array}$




\section{Wireless Temperature Sensing with BST-Based Chipless Transponder Utilizing a Passive Phase Modulation Scheme}

\author{
Christian Mandel, ${ }^{1, *}$ Holger Maune, ${ }^{1}$ \\ Matthias Maasch, ${ }^{1}$ Mohsen Sazegar, ${ }^{1}$ \\ Bernd Kubina, ${ }^{1}$ Martin Schüßler ${ }^{1}$ and Rolf Jakoby ${ }^{1}$ \\ ${ }^{1}$ Microwave Engineering, Technische Universität Darm- \\ stadt, Germany
}

\begin{abstract}
A passive wireless temperature sensor with identification capabilities based on a phase modulation scheme is discussed in this paper. The approach presented utilizes a pulse backscatter technique based on slow wave (metamaterial) transmission lines. The focus of the work are the material engineering for the temperature-sensitive element and the integration of this element into a passive phase modulation circuit and the entire sensor tag. The approach makes use of temperature-sensitive bariumstrontium-titanate thick film capacitances. The discussed principle has been experimentally verified with a prototype.
\end{abstract}

Keywords. Ferroelectric devices, metamaterials, passive microwave remote sensing, radiofrequency identification, sensor systems and applications, temperature sensors.

PACS $^{\circledR}$ (2010). 07.07.Df, 84.40.Xb, 77.84.Cg, 78.67.Pt, 84.40.Dc.

\section{Introduction}

Wireless sensors are emerging in more and more industrial processes. Reasons for using wireless sensors can be the wireless connection being less expensive than a wire connection, or the measured location being not at all accessible by a wire connection. Passive, which means not active powered, wireless sensors are a special subset of these sensors for environments where no additional power supply is available. Hence, these sensors are using energy harvesting techniques or are directly driven by the interrogation signal. Further, chipless wireless sensors refer to a class of designs without digital data processing on the tag side. Especially, no silicon devices are needed on these tags.

Corresponding author: Christian Mandel, Institut für Mikrowellentechnik und Photonik, Technische Universität Darmstadt, Merckstraße 25, 64283 Darmstadt, Germany; E-mail:mandel@imp.tu-darmstadt.de.

Received: June 28, 2011.
Nowadays, most of the passive chipless sensors are done in surface acoustic wave (SAW) technology, which is an electro-mechanical approach of information encoding and backscatter based on a time-domain signature [5]. The sensing capabilities based on this technology are limited to a change in propagation properties of an acoustic wave.

The technology presented in this paper shows an entirely electromagnetic approach of a time-domain-signaturebased wireless sensor buildup with additional identification (ID) opportunity allowing an integration of several sensors into a wireless sensor network.

A separation of tag responses is, unlike the SAW approach, difficult to realize by time slot assignment, since the realizable delays do not permit that. A reasonable opportunity within the presented approach is given by space division multiple access (SDMA), e.g. using a steerable antenna scanning over all sensors in reading range.

The idea behind the time-domain information encoding is sketched in Figure 1. An electromagnetic impulse received by an antenna is travelling along a transmission line (TL) and therefore delayed. Fractions of the pulse are reflected at certain "modulators" in a defined way so that information is encoded. The simplest way of doing this is the reflection vs. no reflection scheme introduced in [2]. The single pulses add up to a pulse-train which is backscattered by the tag's antenna.

This scheme is similar to the one used with SAW tags, a pulse travelling along a waveguide being partially reflected for information encoding. The approach has assets and drawbacks compared to the classic SAW approach. The three main advantages of this technique are the integration of many more different measurement principles, the opportunity of a buildup for very high temperature operation, and the possibility of comparatively cheap buildup/configuration especially at a very low number of devices, e.g. for very specific requirements. Drawbacks are a larger size, and less amount of encodable information.

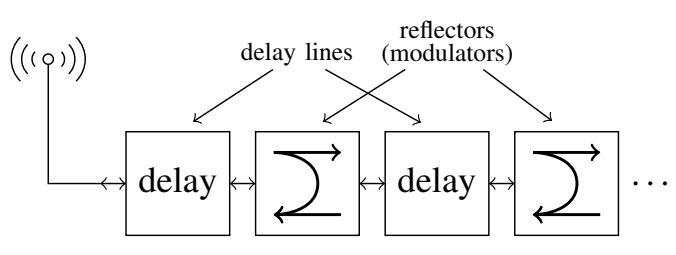

Figure 1. Signal flow in a passive, delay-line-based pulse modulator. 
One key element of the sensor tag is the sensitive element, which can be realized by a temperature-sensitive capacity. The ceramic material barium-strontium-titanate (BST) shows an intrinsic temperature dependency of the material's relative permittivity, which can be tailored in a wide range to an application's needs. Furthermore, the utilization of ceramic materials like BST as temperature sensor has additional benefits: the material itself is sintered at temperatures around $1200^{\circ} \mathrm{C}$, which allows an operation at very high temperatures. The limit for the devices operation temperature range are mainly given by the electrode material.

\section{Slow-Wave (Metamaterial) Delay Line}

First introduced in [2] a metamaterial-based approach of a delayline-based time-domain backscatter modulator allows for comparatively long delays. One key component of the presented approach is the delay line itself which provides for subsequent reflections being distinguishable and therefore determins the necessary bandwidth for the readout of the wireless sensor, basically limited by the Küpfmüller uncertainty principle. The realizable delay is opposed to the attenuation of the line and the maximum overall space on the tag.

To reach a reasonable tag size, a metamaterial approach was chosen. The dispersion diagram of a purely left-handed transmission line, shown in Figure 2, $\omega\left(\beta_{\mathrm{LH}}\right)$, allows the realization of a longer delay compared to its right-handed counterpart. Longest delays are reached at lower frequencies. From this observation, the transmission band for the given purpose is best placed just before the lower lefthanded band edge.

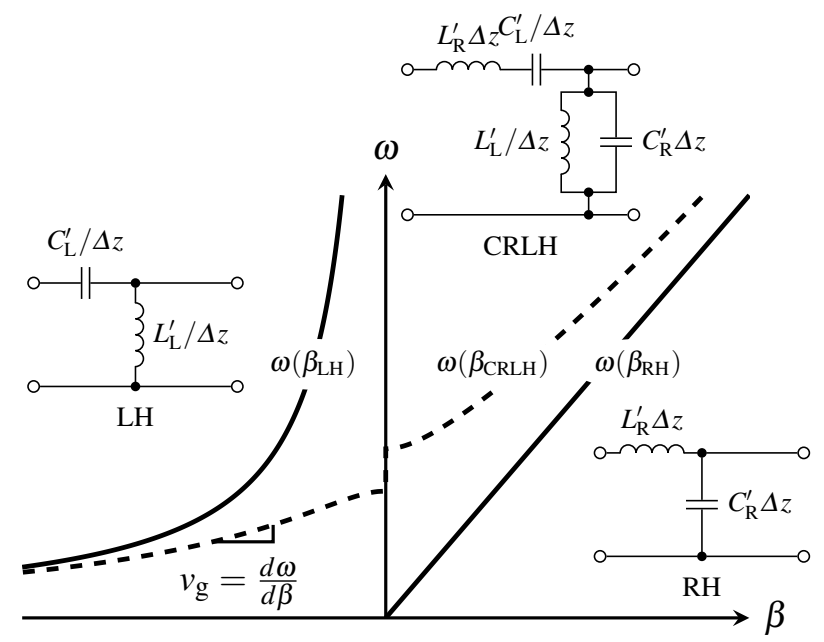

Figure 2. Dispersion diagram of LH, RH and CRLH delay lines and their corresponding lumped element models.
An implementation of a left-handed line shows, due to parasitic effects, always also a right-handed behavior, which leads to the Composite Right/Left-Handed (CRLH) line model, also shown in Figure 2. The parasitic righthanded behavior contributes also to the overall delay, which has to be taken into account in the design process.

As can be also seen from the picture, the metamaterial approach leads to a dispersive behavior of the transmission line, resulting in a pulse broadening over the line. Being a deterministic effect, the dispersion can be compensated at the reader. In praxis, the quality of the compensation result is limited due to the inhomogenity of the realization of the tag. This has also to be taken into account in the design process. Therefore, a realization without dispersion compensation was chosen for the prototype system treated in this paper.

\section{Passive Phase Modulation}

The passive phase modulation/phase shift keying, first introduced in [3], allows for an increased information density, analog to the phase modulation schemes widely used in mobile communications and wireless networks. In the passive chipless domain a phase modulation can be achieved by introducing a modulator circuit realizing not only a fractional amplitude backscatter but also a phase shift of the carrier. For a sensor, in most cases a continuous coverage of a range of sensable values would be preferred, meaning an analog phase modulation dependent on the sensed physical parameter, e.g. temperature.

Figure 3 shows an overview over the components of a passive chipless sensor tag implementing a temperature sensor using the techniques described before.

The dependence of the phase shift of the reflected signal caused by the sensor element on the sensed physical param-

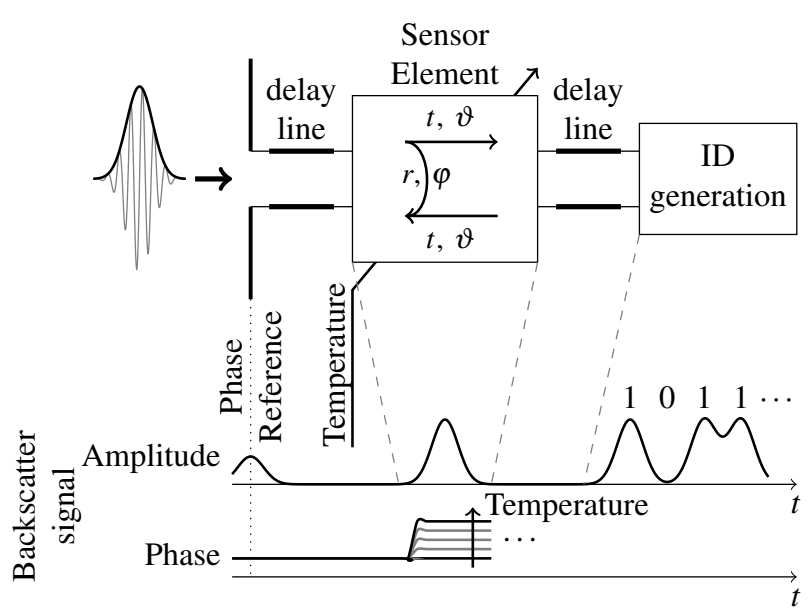

Figure 3. Structure and operation principle of a passive sensor tag phase-coding the sensed value (temperature). 
eter should be designable. A phase reference on the tag is necessary since the absolute received phase depends on the reading distance and the environment of the tag (and the reader). If the tag's antenna is not influenced by the environment, the direct backscatter signal of the antenna may be used for this purpose (as drawn in Figure 3), but also a particularly generated reference pulse on the line structure (before or behind the sensor) is possible.

The transmission phase shift of the sensor has to be compensated in case of a passive phase-shift keying (PSK) ID generation, since every ID pulse will pass the sensor two times, what will change its phase. A low temperature dependence of this phase shift can therefore simplify the information decoding process and reduce decoding errors.

The sensor has to meet a second requirement, even if a simple on-off pulse modulator is used for ID generation: The pulse energy transmitted through the sensor element has to drive the ID generation while the reflected pulse encoding the sensed value has to obtain enough energy to assure a reasonable signal quality at the reader. The magnitude of the reflection and transmission coefficient have therefore to be designed to stay within reasonable boundaries.

Different modulator structures have been evaluated, as can be seen later. The temperature dependent part of the phase shifter used is realized by an interdigital capacitor based on BST thick film technology, since the BST material shows an intrinsic temperature dependence of the permittivity and thus, of the capacitance of the device.

\section{BST Capacitors as Temperature Sensitive Element}

The presented sensor concept utilizes the change of the capacitance of the sensing elements. For high temperature sensors only few materials such as ceramics are useable. Barium-Strontium-Titanate, $\mathrm{Ba}_{x} \mathrm{Sr}_{1-x} \mathrm{TiO}_{3}$ known from tunable microwave components, can be specifically engineered to fulfill the requirements for temperature sensing in a wide temperature range. Starting from the Landau theory, Ginzburg and Devonshire developed a phenomenological description of the ferroelectric phase transition resulting in the temperature dependent relative permittivity [6]

$$
\begin{aligned}
& \varepsilon_{r}(T)= \\
& \frac{\varepsilon_{00}}{\left(\sqrt{\xi_{S}^{2}+\eta^{3}}+\xi_{S}\right)^{\frac{2}{3}}+\left(\sqrt{\xi_{S}^{2}+\eta^{3}}-\xi_{S}\right)^{\frac{2}{3}}-\eta+\underline{\Gamma}}
\end{aligned}
$$

with

$$
\eta(T)=\sqrt{\left(\frac{\Theta_{F}}{4 T_{C}}\right)^{2}+\left(\frac{T}{T_{C}}\right)^{2}}-1
$$

with the external temperature $T$, Curie temperature $T_{C}$, Curie constant $\varepsilon_{00}$, Debye temperature $\Theta_{F}$, and the material quality $\xi_{S}$. The losses are summarized in $\underline{\Gamma}(T, \omega)$. The model is well suited in the vicinity of the tetragonal/cubic phase transition $\left(T \approx T_{C}\right)$ and within the paraelectric region $\left(T>T_{C}\right)$, the region of interest for applications. The Curie temperature and hence the area of operation can be adapted by the ratio of Barium to Strontium in $\mathrm{Ba}_{x} \mathrm{Sr}_{1-x} \mathrm{TiO}_{3}$ [1], and it can be approximated by

$$
T_{C}(x)=\left[42+439.37 \cdot x-95.95 \cdot x^{2}\right] \mathrm{K} .
$$

For $x=0.6$ the Curie point is at approximately $-2{ }^{\circ} \mathrm{C}$, making the material usable for room temperature and above.

Figure 4 shows the theoretical temperature dependency of the permittivity for different material quality factors and $\mathrm{Ba} / \mathrm{Sr}$ ratios and a measurement at two different frequencies. BST bulk ceramics $\left(\xi_{S} \approx 0.01\right)$ show a very high

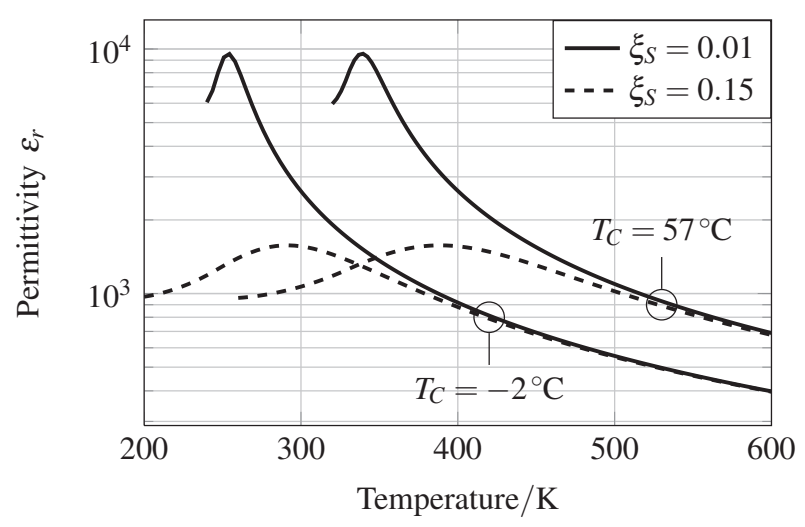

(a) Temperature dependency for different material quality factors $\xi_{S}$ and different $\mathrm{Ba} / \mathrm{Sr}$ ratios $x$. The material parameters are chosen to be $\varepsilon_{00}=560$ and $\Theta_{F}=175 \mathrm{~K}$. The Curie temperature $T_{C}$ is calculated according to (3).

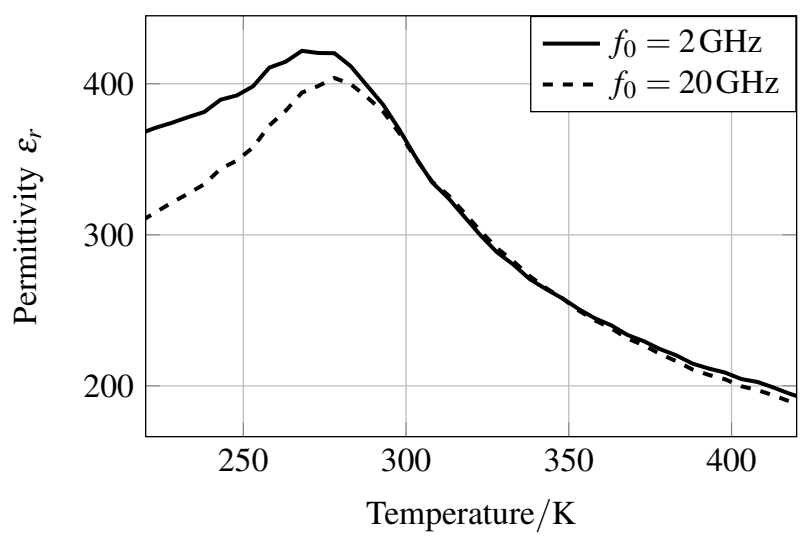

(b) Measured temperature dependency of a BST thick film (deembedded CPW measurement) with material composition ratio $x=0.6, T_{C} \approx-2{ }^{\circ} \mathrm{C}$, at different frequencies.

Figure 4. Temperature dependency of the permittivity of BST, (a) from theory and (b) measured. 


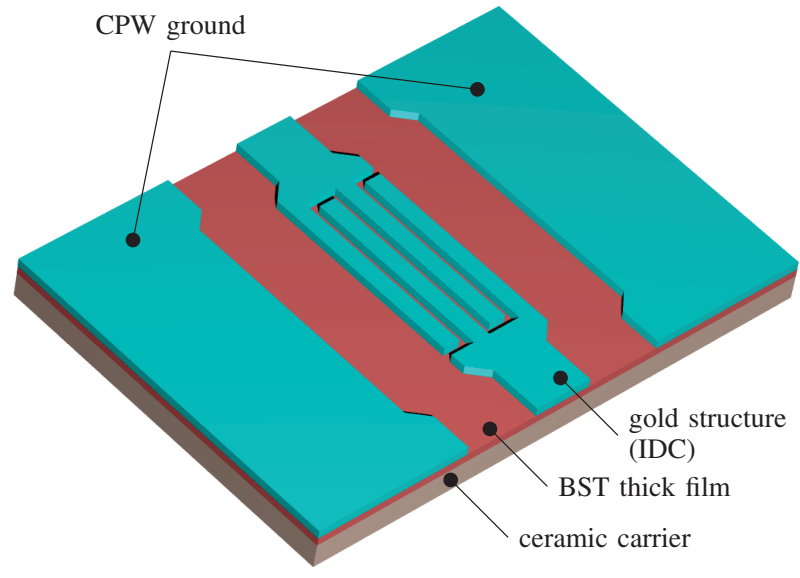

Figure 5. Inter-digital capacitor build-up on BST thickfilm. The CPW ground shown here is used for characterization, this part is not present in the sensor element.

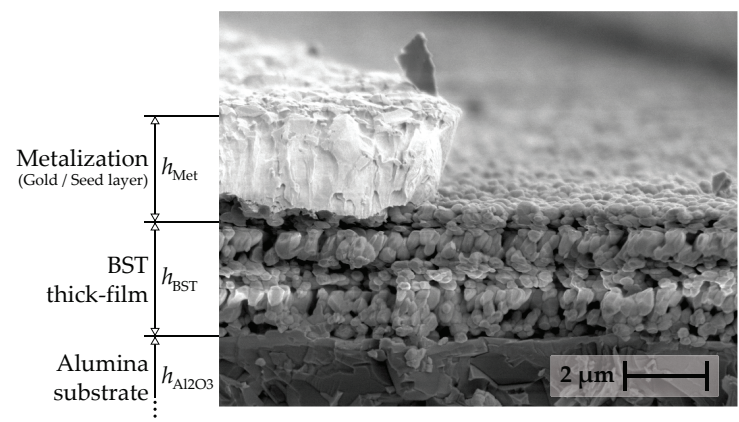

Figure 6. Scanning electron microscope picture of the cross section of a tunable component based on BST thick film technology.

temperature coefficient in the vicinity of the phase transition compared to BST thick films $\left(\xi_{S} \approx 0.15\right)$. Different $\mathrm{Ba} / \mathrm{Sr}$ ratios can be used to adapt the area of application to different needs, in particular for high temperature applications. The temperature dependency can be adapted to the needs by doping the material or changing the processing technology, in order to change the Curie temperature and the material quality.

Based on these facts BST used in capacitors is a suitable material for temperature sensing. The capacitors are built as interdigital capacitors (IDC) on top of a screen printed and sintered thick film. Figure 5 shows the IDC build-up schematically and a cross section scanning electron microscope picture of a BST thick-film IDC build-up is shown in Figure 6.

The alumina substrate usually has a thickness of $h_{\mathrm{Al}_{2} \mathrm{O}_{3}}=650 \mu \mathrm{m}$. The thickness of the BST thick film and the metallization is optimized for the component design [4] and varies between $h_{\mathrm{BST}}=2 \ldots 10 \mu \mathrm{m}$ and $h_{\mathrm{Met}}=$ $2 \ldots 5 \mu \mathrm{m}$, respectively. The change of the capacitance value $\Delta C(T)$ is related to the change of the permittivity
$\Delta \varepsilon$ by

$$
\Delta C=\chi_{\mathrm{geo}} \cdot \Delta \varepsilon
$$

where $\chi_{\text {geo }}$ is the geometric efficiency of the capacitor design, especially the relation between gap width of the IDC and the height of the BST thick film.

\section{Prototype Realization and Results}

For an independent verification of the components of the entire sensor tag, the prototype of the ID modulating part and the sensor part have been independently realized, as can be seen in Figure 7.

Wireless and wired measurements were carried out by the utilization of a vector network analyzer (VNA), implementing a FMCW-like (frequency modulated continuous wave) readout. The measured frequency domain response was transformed to the time domain by means of Fourier transform, leading to a desired time-domain signal. A wireless measurement setup is shown in Figure 8.

Figure 9 shows simulation and measurement results of the input reflection and transmission of the delay-line used in the prototype. The line consists of three sections (each section consists of 20 cells), each providing a two-way delay of about $5 \mathrm{~ns}$. This value determins the boundaries of the read-out parameters, as described before.

The marked transmission band is placed just above the lower left-handed band edge, as suggested in section 2, where the delay over loss ratio becomes best. The overall loss for a one-way transmission is in the range of $4 \mathrm{~dB}$, approx. $1.5 \mathrm{~dB}$ higher than simulated.

For the sensor a circuit was chosen which fulfills the requirements high sensitivity (which is equivalent to a high differential phase shift) in reflection, low amplitude variation, and easy integration into the delay line structure. Figure 10 compares three unit cell structures realizing a capacitive sensor. The input reflection coefficient is plotted

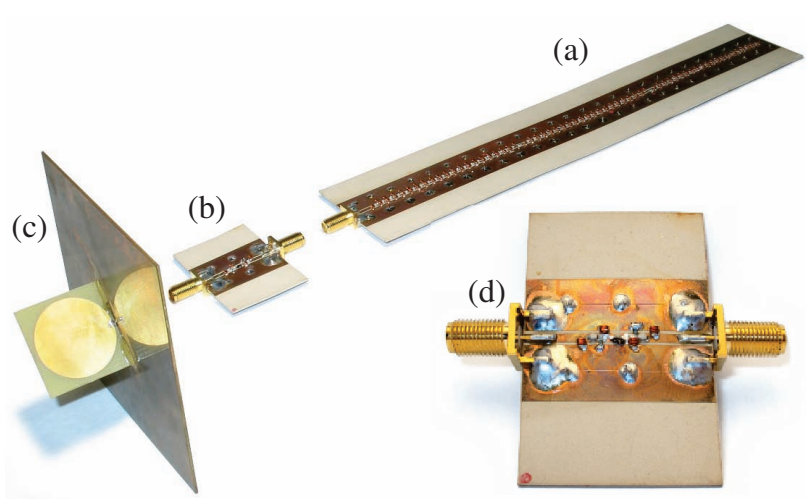

Figure 7. Photographs of the prototypes: (a) RFID modulator, (b) BST temperature sensor, and (c) broadband monopole antenna. (d) shows a closer view of (b). 


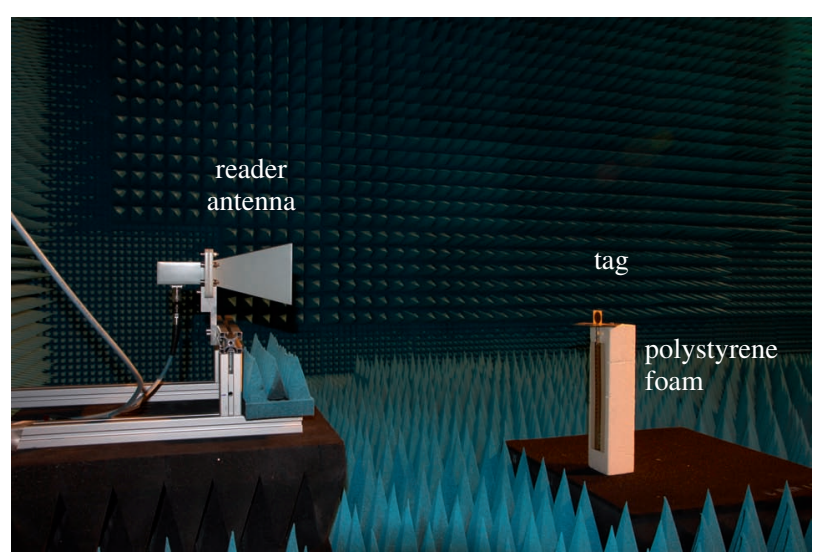

Figure 8. Wireless read-out setup, showing the ID tag on a polystyrene foam mounting. The whole setup is placed inside of an anechoic chamber.

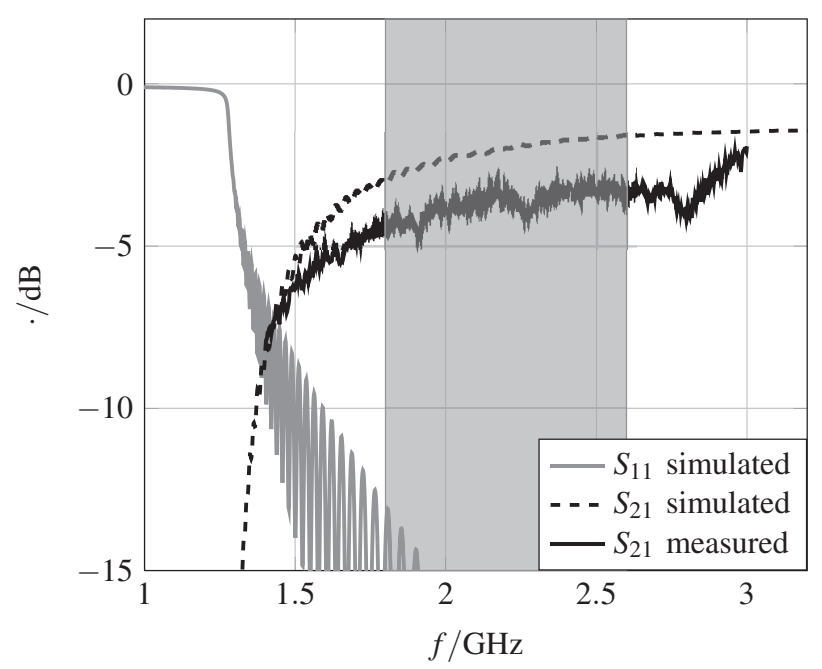

Figure 9. Simulation and measurement results of a 60-cell metamaterial transmission line as used for the prototype. The gray marked area highlights the band used for the sensor and ID read-out.

for these structures being terminated with $50 \Omega$. The goal for all structures was an approximation of a section of the $|r|=0.3$ circle. Using a series capacitor leads to a $19^{\circ}$ phase shift with large magnitude variation. The L network approach shows almost no magnitude variation and a higher phase shift of $39^{\circ}$. The largest phase shift of $143^{\circ}$ can be achieved with the asymmetric network, but again introducing a magnitude variation. For high sensitivity, the third is clearly the best fitting approach, so it was chosen for the realized prototype.

Figure 11 shows simulation results of the final prototype design for single-frequency excitation at carrier frequency and band edges of the read-out signal. The design goal was given by a reflection magnitude lying between 0.1 and 0.3 for the whole read-out band to maintain enough pulse

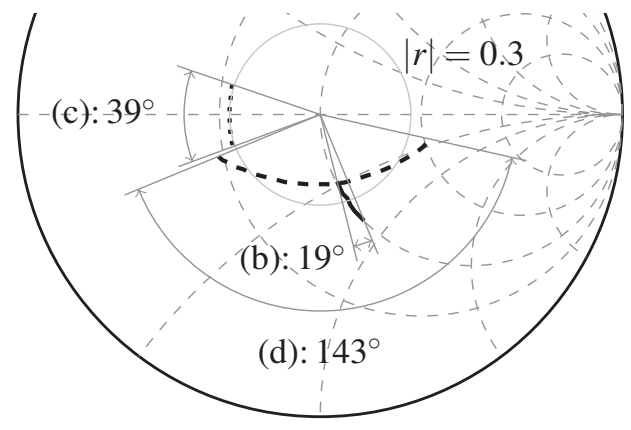

(a) reflection properties of the circuits below

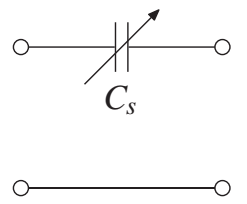

(b) series capacitor

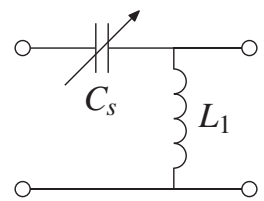

(c) L network

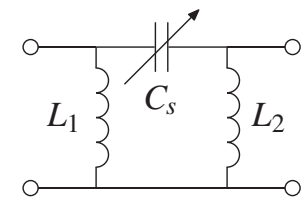

(d) $\Pi$ network
Figure 10. Comparison of different modulator cells for passive analog phase modulation with capacitances dependent on the sensed property (capacity change: $\pm 25 \%$ ).

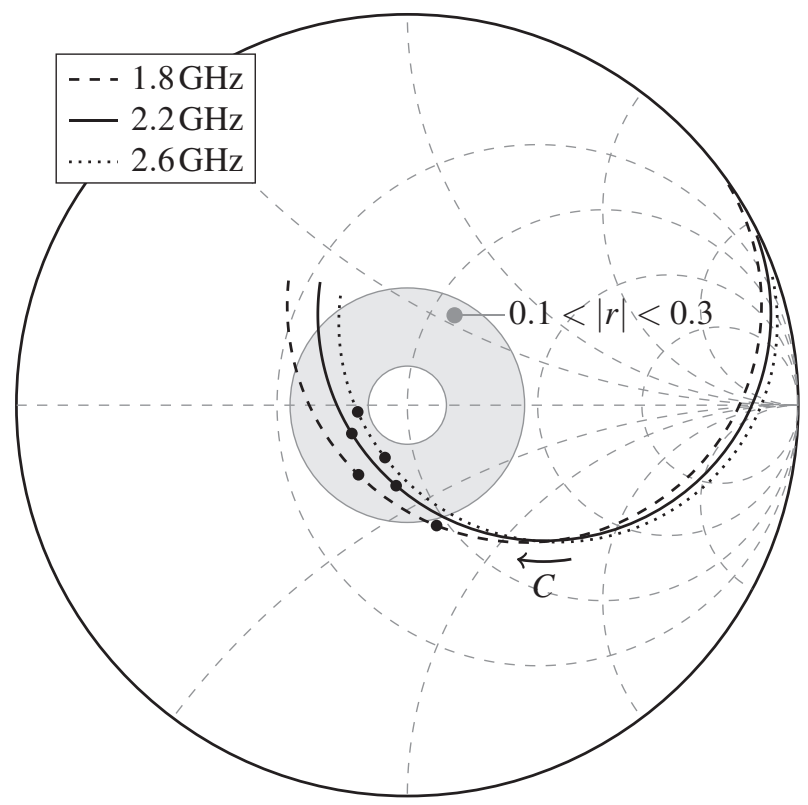

Figure 11. Construction goals and simulations of the sensor element at center frequency and band edges. The dots on the lines mark the expected tuning range of the BST capacitance from $C_{0}$ to $C_{0}-25 \%$.

energy for the ID generation but also have a reasonable backscatter magnitude for the sensed value. The goal is almost met, the slight violation at the lower band edge is caused by the discrete nature of available SMD component values.

As an additional benefit, it also shows a low phase change in transmission direction. 


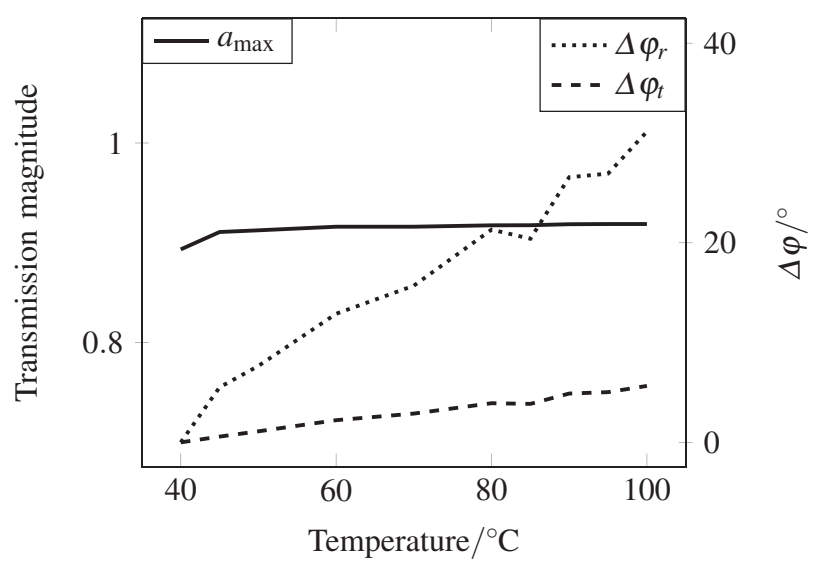

Figure 12. Measured transmission and reflection amplitude and phase change of the sensor over temperature.

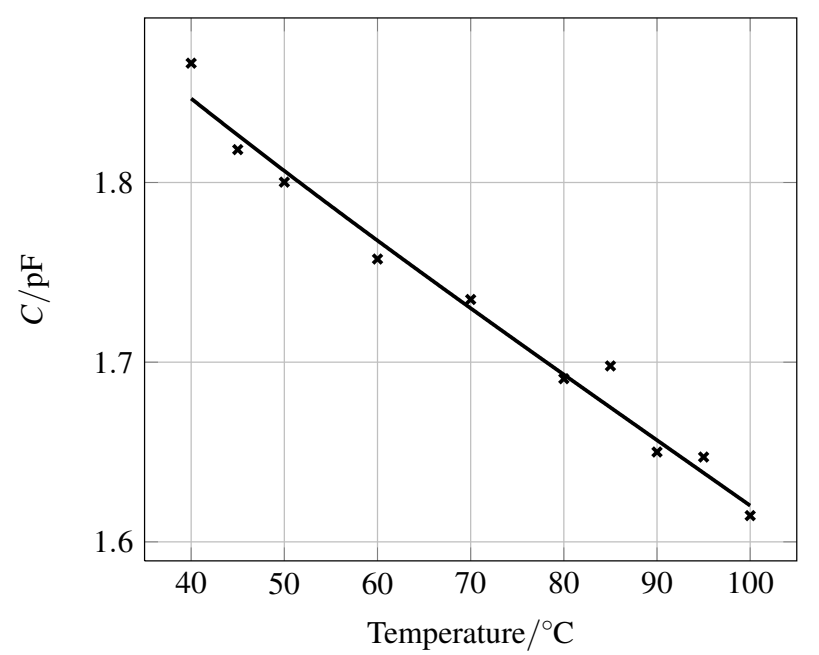

Figure 13. Capacity of the sensing BST capacitor over temperature calculated from the phase measurement data and the lumped element model of the sensor.

These findings can also be observed in the measurement results, as shown in Figure 12, which were obtained using a raised-cosine shaped pulse with a bandwidth of $B=800 \mathrm{MHz}$ and a carrier frequency of $f_{c}=2.2 \mathrm{GHz}$, measured as described before.

The amplitude curve shows the amplitude transmission of the device, which indicates only a slight change over temperature. Since the sensor circuit can be considered as nearly lossless, the reflection amplitude shows a similar behavior.

From the phase plot, a high sensitivity of the sensor can be observed, achieving about $0.5^{\circ} / \mathrm{K}$. The transmission phase shift is, as mentioned before, much lower, about $0.1^{\circ} / \mathrm{K}$.

Figure 13 shows the capacitance of the BST capacitor over the temperature. This data points have been calculated using the measured differential phase shift of the sen-

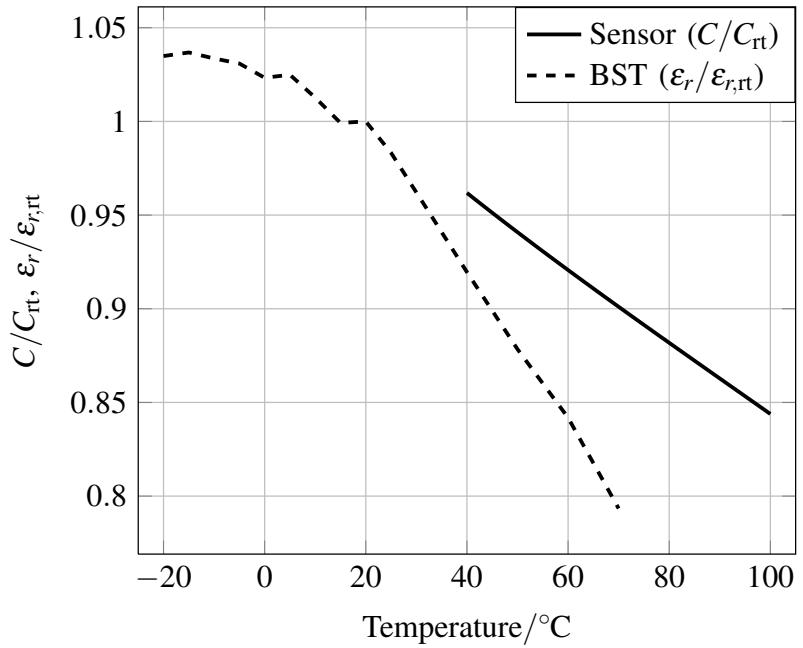

Figure 14. Comparison of the temperature behavior of the BST capacitor resp. material in terms of capacity resp. permittivity (rt: room temperature, $21^{\circ} \mathrm{C}$ ).

sor and a lossy series resonance lumped element model of the capacitor. The curve shows the linear regression over the measured points. This result is in good agreement with the material considerations from the previous section, assuming an operation in the almost linear region above the Curie temperature.

The comparison of the resulting regression line and the BST material measurement of Figure 14 seconds this observation. The difference in the slope of the curves is caused by the device parasitics not regarded in the material curves and the comparatively simple capacitor model.

\section{Conclusion and Outlook}

This paper presents a BST-based ID-equipped passive chipless wireless sensor and gives an overview over material engineering as well as circuit design. The introduced concept can be easily adapted to ceramic substrates and a substratefocused design aiming for sensors operating at very high temperatures. The same holds in principle for the BST material. A shift to higher frequencies can shrink the size of the tag and ease the application of SDMA.

For the permittivity of BST being not only dependent on the temperature, also other sensor concepts are conceivable, even concepts introducing active layers allowing e.g. gas concentration detection.

\section{References}

[1] Jae-Ho Jeon, Effect of SrTiO3 concentration and sintering temperature on microstructure and dielectric constant of Ba1-xSrxTiO3, Journal of the European Ceramic Society 24 (2004), 1045-1048. 
[2] Martin Schüßler, Christian Damm, Matthias Maasch and Rolf Jakoby, Performance Evaluation of Left-Handed Delay Lines for RFID Backscatter Applications, in: Proc. of the IEEE MTT-S International Microwave Symposium, 2008.

[3] Christian Mandel, Martin Schüßler, Matthias Maasch and Rolf Jakoby, A Novel Passive Phase Modulator based on LH Delay Lines for Chipless Microwave RFID Applications, in: IEEE MTT-S International Microwave Workshop on Wireless Sensing, Local Positioning, and RFID, 2009.

[4] H. Maune, M. Sazegar, Y. Zheng, X. Zhou, A. Giere, P. Scheele, F. Paul, J. R. Binder and R. Jakoby, Nonlin- ear Ceramics for Tunable Microwave Devices Part II: RFCharacteriziation and Component Design, Microsystem Technologies 17 (2011), 213-224.

[5] A. Pohl, A review of wireless SAW sensors, IEEE Transactions on Ultrasonics, Ferroelectrics and Frequency Control 47 (2000), 317-332.

[6] O. G. Vendik and S. P. Zubko, Ferroelectric phase transition and maximum dielectric permittivity of displacement type ferroelectrics (BaxSr1-xTiO3), Journal of Applied Physics $\mathbf{8 8}$ (2000), 5343-5350. 